\title{
Exploring Blink-Rate Behaviors for Cybersickness Detection in VR
}

\author{
Phil Lopes* \\ EPFL \\ Lausanne, Switzerland
}

\author{
Nana $\operatorname{Tian}^{\dagger}$ \\ EPFL \\ Lausanne, Switzerland
}

\author{
Ronan Boulic \\ EPFL \\ Lausanne, Switzerland
}

\begin{abstract}
Cybersickness continues to be one of the main barriers for mainstream adoption of Virtual Reality (VR). Despite the wealth of research available on this topic, it is still an unsolved problem. This paper explores the potential influence of cybersickness over the course of a VR experience on player Blink-Rate. This investigation was conducted on the same VR task with two diverging control schemes with a low or high risk of inducing cybersickness. A total of 34 participant data was collected from two separate playing sessions. Although no significant differences were observed, sick individuals showed to have a higher blink-rate frequency over the course of the VR experience while non-sick individuals presented a more stable blink-rate frequency.
\end{abstract}

Index Terms: Human-centered computing-Human Computer Interaction (HCI) - Interaction Paradigms - Virtual Reality; Humancentered computing-Human Computer Interaction (HCI)-HCI design and evaluation methods-User Studies

\section{INTRODUCTION}

Despite the popularity of Virtual Reality (VR) over the past years, VR-induced sickness still remains a serious issue that can severely hinder the experience of its users, consequently limiting its widespread adoption [12]. Although a wide variety of research has been conducted on this topic, no specific explanation currently exists on what exactly contributes to this phenomena. The most popular theory includes the visual-vestibular conflict [8] and visually-induced self-motion illusion [1]. However, the literature often presents conflicting results when it comes to pin-pointing the exact causes of cybersickness. Thus, making a generic solution capable of combating this problem difficult to achieve considering that the personal factors of each individual can also determine this risk.

\section{Related Work}

Previous literature suggests that one method of detecting cybersickness is to analyze human bodily responses - obtained through physiological sensors $[3,7]$. Given the influence of cybersickness to human bodily functions it comes as no surprise that physiological measures have often been employed for its detection and prediction. Although the majority of the correlations between the physiological data and self-assessed responses of cybersickness were found to be inconclusive - using the Simulation Sickness Questionnaire [6] - one of the more promising factors was eye-tracking (i.e. Blink-Rate and Pupil Position).

Blink rate has often been associated with negative human behaviours such as stress and fatigue [9]. Furthermore, empirical studies have also suggested that changes in blink rate can also be a reliable measure for eyestrain [7]. Given that eye fatigue is one of the core symptoms of the SSQ [6] it makes sense to explore this factor as a potential variable for cybersickness detection [4].

\footnotetext{
*e-mail: phil.lopes@epfl.ch

†e-mail: nana.tian@epfl.ch

‡e-mail: ronan.boulic@epfl.ch
}

This work intends to further explore the eye blink frequency phenomenon on the effects of cybersickness. Unlike previous work, which exclusively explored the correlation between SSQ and blink rate, this paper focuses on comparing the blink rate between cybersick and non-cybersick individuals and infer if there is a difference between both populations.

\section{Methodology}

Purposefully inducing cybersickness is a delicate process as the tolerance can vary substantially between individuals. Thus, to conduct such an experiment with a degree of success, an extensive piloting phase was conducted. Based on these empirical tests, two alternate versions of the game were developed, where one version of the game was designed to have a higher risk of inducing cybersickness than the other.

\subsection{Apparatus}

The head-mounted display used in this study is the HTC Vive Pro Eye (HTC, 2019). Both eyes update data at the sampling rate of $120 \mathrm{HZ}$, and the estimated accuracy of the eye tracker is 0.5 degrees with a latency of approximately $10 \mathrm{~ms}$.

\subsection{The Game}

The task consists in an exploration game where players must collect as many coins as possible within a large virtual maze over a period of time. Coins are placed in such a way that it constantly pushes the player to move forward ${ }^{1}$. Lastly, each participant plays a level for a total of 8 minutes.

Movement within VR environments is one of the main contributors of cybersickness [13], as the dissonance between virtual rotation and the lack of physical movement can significantly conflict with the vestibular system [10]. Two variants of the game were developed with different types of player displacement mechanics: the Teleportation (TP) and Standard Control (SC).

\subsubsection{Teleportation Control (TP) - Low Risk}

This control scheme has players point to a location within the virtual environment and choose which location they wish to move to. Previous research has suggested that "teleportation-type" control schemes tend to provide comfortable experiences and provide less risk of inducing cybersickness [13]. Thus, this control scheme is designed to be the "low-risk" variation for inducing cybersickness.

\subsubsection{Standard Control (SC) - High Risk}

This control scheme uses exclusively the two joysticks available on the controller. The left joystick controls the player displacement, while the right joystick allows the players to rotate their virtual avatar. Unlike teleportation, this control scheme is often avoided due to its high risk of inducing cybersickness during actual play [2]. As such, this control scheme is purposefully designed to be the "higher-risk" variation.

\subsection{Blinking Data Markers}

For the purpose of this work three types of blinks are tracked and classified according to [11] as such: Short Blink (1 to $7 \mathrm{~ms}$ ), Medium Blink ( 8 to $15 \mathrm{~ms}$ ) and Extended Blink (16 to $25 \mathrm{~ms}$ ). 


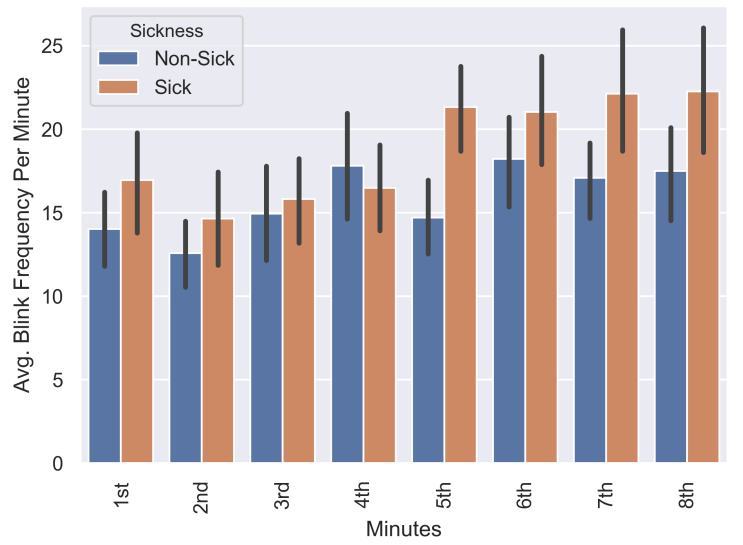

Figure 1: The Average and Standard Error of the Blink Frequency per Minute across all participants for both Sick and Non-Sick Groups.

\subsection{Simulation Sickness Questionnaire (SSQ)}

To assess the intensity of cybersickness among participants the standard Simulation Sickness Questionnaire (SSQ) was employed [6]. The SSQ can derive a rough numerical estimate of the intensity of cybersickness felt by each individual. To obtain a more consistent representation a pre-SSQ is filled before the start of the experiment while the post-SSQ is filled at the end of the experiment. The difference between the post- and pre-SSQ is subsequently calculated $(\Delta \mathrm{SSQ})$.

\subsection{Experimental Protocol}

The experiment takes place over the course of two different sessions of around 40 minutes each. Each session is conducted on two different days (with a maximum interval of $\approx 1$ to 3 days). For each session a participant plays one variation of the game (SC or TP). To ensure an equal balance between both variations and mitigate an "order"-bias, the first version played by each participant alternates from one participant to the next. It is important to keep in mind that all participants play both variations of the game. Each session employs the same sequence.

\section{Results}

In total 36 participants were recruited for the experiment. Out of the 36 participants 2 participants had to be fully discarded due to one abandoning the experiment midway and another presenting corrupted data. As a result a total of 34 participants presented valid sessions for both SC and TP. Based on the SSQ results, each session was distributed into two categories: $\operatorname{Sick}(\triangle S S Q \geq 15)$ and Non-Sick $(\triangle S S Q<15)$ for a total of 32 and 36 sessions in each respective category. This value was chosen based on previous literature [5], which states that scores above this value are "sufficiently" high enough to feel the effects of motion sickness.

The average blink rate per minute was plotted across all participants for both sick and non-sick conditions (see Fig. 1). A KS-Test was also conducted to verify if there was a significant difference between the blink-rate of both groups, which yielded a $p_{\text {value }}$ of $(\approx 0.28)$ still above the standard 0.05. Although inconclusive, it can be observed that the blink-rate frequency tends to increase for sick individuals comparatively to non-sick. This does go inline with previous literature where a correlation was observed between cybersickness and blink rate [7]. Given that the blink frequency per minute tends to extensively increase in the latter stages of the game.

\section{Conclusions}

Early results show an interesting pattern for "short blinks" specifically. Although the difference between Sick and Non-Sick are not statistically significant an interesting pattern did emerge when observing the blink-rate of both groups. Sessions where individuals reported to be sick tended to have a higher blinking frequency per minute than non-sick sessions.

\section{ACKNOWLEDGMENTS}

This research was supported by the SNF grant CRSII5_180319 / 1.

\section{REFERENCES}

[1] M. Al Zayer, I. B. Adhanom, P. MacNeilage, and E. Folmer. The effect of field-of-view restriction on sex bias in vr sickness and spatial navigation performance. In Proceedings of the 2019 CHI Conference on Human Factors in Computing Systems, CHI '19, pp. 354:1-354:12. ACM, New York, NY, USA, 2019. doi: 10.1145/3290605.3300584

[2] M. Al Zayer, P. MacNeilage, and E. Folmer. Virtual locomotion: a survey. IEEE Transactions on Visualization and Computer Graphics, PP:1-1, 12 2018. doi: 10.1109/TVCG.2018.2887379

[3] S. Davis, K. Nesbitt, and E. Nalivaiko. A systematic review of cybersickness. In Proceedings of the 2014 Conference on Interactive Entertainment, pp. 1-9. ACM, 2014.

[4] J. Iskander, M. Hossny, and S. Nahavandi. A review on ocular biomechanic models for assessing visual fatigue in virtual reality. IEEE Access, 6:19345-19361, 2018.

[5] R. S. Kennedy, J. M. Drexler, D. E. Compton, K. M. Stanney, D. S. Lanham, and D. L. Harm. Configural scoring of simulator sickness, cybersickness and space adaptation syndrome: Similarities and differences. Virtual and adaptive environments: Applications, implications, and human performance issues, p. 247, 2003.

[6] R. S. Kennedy, N. E. Lane, K. S. Berbaum, and M. G. Lilienthal. Simulator sickness questionnaire: An enhanced method for quantifying simulator sickness. The international journal of aviation psychology, 3(3):203-220, 1993.

[7] Y. Y. Kim, H. J. Kim, E. N. Kim, H. D. Ko, and H. T. Kim. Characteristic changes in the physiological components of cybersickness. Psychophysiology, 42(5):616-625, 2005.

[8] J. J. LaViola, Jr. A discussion of cybersickness in virtual environments. SIGCHI Bull., 32(1):47-56, Jan. 2000. doi: 10.1145/333329.333344

[9] E. C. Lee, H. Heo, and K. R. Park. The comparative measurements of eyestrain caused by $2 \mathrm{~d}$ and $3 \mathrm{~d}$ displays. IEEE Transactions on Consumer Electronics, 56(3):1677-1683, 2010.

[10] M. E. McCauley and T. J. Sharkey. Cybersickness: Perception of self-motion in virtual environments. Presence: Teleoperators \& Virtual Environments, 1(3):311-318, 1992.

[11] G. W. Ousler 3rd, M. B. Abelson, P. R. Johnston, J. Rodriguez, K. Lane, and L. M. Smith. Blink patterns and lid-contact times in dry-eye and normal subjects. Clinical ophthalmology (Auckland, NZ), 8:869, 2014.

[12] L. Rebenitsch and C. Owen. Review on cybersickness in applications and visual displays. Virtual Reality, 20, 04 2016. doi: 10.1007/s10055 -016-0285-9

[13] T. Weißker, A. Kunert, B. Fröhlich, and A. Kulik. Spatial updating and simulator sickness during steering and jumping in immersive virtual environments. In 2018 IEEE Conference on Virtual Reality and $3 d$ User Interfaces (VR), pp. 97-104. IEEE, 2018. 